\title{
- Latest Genetic Diagnostic Technologies
}

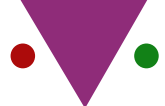 \\ IJCRR \\ Section: Healthcare \\ ISI Impact Factor \\ (2019-20): 1.628 \\ IC Value (2019): 90.81 \\ SJIF (2020) $=7.893$ \\ (c) (2) (9) \\ Copyright@IJCRR

\section{Anshda Bhatnagar} \\ Department of Health and Life Sciences, Coventry University, Coventry $\mathrm{CV}_{15} \mathrm{FB}$, United Kingdom.
}

\section{INTRODUCTION}

Technologies for genetic diagnosis are experimental techniques used to recognise and analyse the genes of an individual. Genes are segments of deoxyribonucleic acid (DNA) that bear a code for a specific protein that functions in the body in one or more cell types. Technology for genetic testing is rapidly developing. To replicate parts of a gene or find changes in genes, different methods can be used. It is a fact that these genetic diagnostic techniques become affordable for individuals to obtain accurate information about their genotype (the particular combination of genes or genetic makeup of a person) in the near future.

\section{GENE PROBE}

Proceeding faster into the field of Molecular Biology and clinical medicine, genetic diagnostic technology called gene probe can be generated in various ways and fall into three categories in general: oligonucleotide probe, polymorphic probes and gene-specific probes. The diagnostic tool can be utilized to locate a specific part of a gene (a segment of the gene's DNA) or a whole gene in a particular chromosome. Probes can be used to find normal or mutated segments of DNA. A DNA segment that has been cloned or copied becomes a labelled probe when a radioactive atom or fluorescent dye is added to it. The probe will seek out its mirror-image segment of DNA and bind to it. Sophisticated microscopic and photographic techniques may then detect the labelled probe. A variety of diseases may be identified pre- and post-birth with gene probes. Gene probes will likely be used in the future to screen individuals concurrently for several significant genetic disorders.

\section{MICROCHIPPING}

With the technology getting under the skin, microchipping is an important instrument to detect DNA mutations, bits of ribonucleic acid (RNA), or proteins that can be used. By using only one sample, a single chip can monitor for millions of different DNA changes.

\section{POLYMERASE CHAIN REACTION}

Another prospecting technique in genetics is Polymerase Chain Reaction (PCR), this is a simple molecular technique used in an exponential way to amplify target sequences from a DNA template making the gene much easier to research. A particular deoxyribonucleic acid (DNA) fragment in a laboratory, such as a specific gene, may be copied (amplified). Starting with one DNA molecule, about a billion copies are generated at the end of 30 duplications (only a few hours later).

\section{OLIGONUCLEOTIDE ARRAYS}

A chain of bases is an oligonucleotide (nucleotides). These chains are often incomplete or have duplicate DNA segments. To locate the deleted or duplicated parts of DNA in particular chromosomes, an oligonucleotide array is used. DNA from a person in an oligonucleotide array is compared to a reference genotype (the particular combination of genes or genetic makeup of a person) using several oligonucleotides probes. A fluorescent dye is applied to the oligonucleotide probes, like other gene probes. The probes detect a reduced amount of the fluorescent dye if a section is missing. If a section is duplicated or tripled, the probes' elevated volume of the fluorescent dye is measured. For evaluating the entire genotype, these probes can be used.

\section{NEXT GENERATION SEQUENCING}

Next-generation sequencing (NGS) is a high-throughput technique that allows the base pairs in RNA and DNA samples to be sequenced rapidly. By splitting the whole genotype (or genome) into small fragments and then examining 
the DNA sequence of some or all of the segments, you can detect even smaller sections of genes and DNA. A robust computer then analyses the findings. As well as places where bases are absent or have been placed in the wrong place, single or multiple variations in bases can be found. The cost of this technology has dropped drastically and continues to decrease. In addition, equipment and computational approaches continue to enhance.Doctors may help diagnose genetic disorders with any of these variants. The next decade's sequencing technologies are so sensitive that doctors can detect DNA from the foetus in a mother's blood sample and examine it to determine whether the foetus has Down syndrome. However, the sheer amount of data produced by genotype analysis results in a number of issues that often make it difficult for doctors to understand and interpret the results. These approaches tend to be the technology of the future, despite these problems.

\section{REFERENCE}

1. David N. Finegold, Genetic Diagnostic Technologies, https:// www.msdmanuals.com/home/fundamentals/genetics/geneticdiagnostic-technologies, accessed on Jan 15, 2021 